\title{
Silicone Band in the Surgical Treatment of Manifest Exotropia
}

\section{Lelio Sabetti, Antonio Berarducci, Domenico Di Lodovico}

Eye Clinic, San Salvatore Hospital, University of L'Aquila, L'Aquila, Italy.

Email: studiosabetti@yahoo.it

Received June $14^{\text {th }}, 2012$; revised August $15^{\text {th }}, 2012$; accepted August $23^{\text {rd }}, 2012$

\begin{abstract}
Purpose: A silicone band was sutured anteriorly to the edge of the lateral rectus muscle neotendon during recession surgery in order to reduce and delay the reoccurrence after exotropia surgery. Methods: Collected data of 12 patients $(8$ males and 4 females), aged 7 - 54 years with diagnosis of constant manifest exotropia and mean angle deviation in primary position of $15,04^{\circ}(\mathrm{sd} 1,18)$ for near and of $13,23^{\circ}(\mathrm{sd} 0,71)$ for distance. All patients underwent surgical treatment consisting of lateral rectus recession and silicone band apposition in correspondence to the anterior edge of the lateral rectus muscle neotendon. Results: A substantial reduction of the mean angle deviation was observed in all patients after surgery. No adverse band-related effects were detected. Conclusion: The silicone band in the surgical treatment of manifest exotropia could be a valuable aid in delaying the relapse thanks to its physical and structural features and to its biocompatibility.
\end{abstract}

Keywords: Silicone Band; Exotropia; Recession; Lateral Rectus Neotendon

\section{Introduction}

Surgery represents the gold standard treatment in eyes exodeviations. Several processes are implicated in the exodeviation genesis but neurological lesions [1] are the most frequent. Surgical technique is planned according to deviation grade, sensory status and age of the patients $[2,3]$ and it includes bilateral lateral rectus recession and/ or unilateral medial rectus resection (which is strictly related with the primary deviation degree [4-6]). The use of adjustable sutures might represent an effective aid in the surgical management [7] as well as dominant or non-dominant eye surgery might produce different functional outcomes $[8,9]$.

Early post surgery complication is represented by surgical hypocorrection [10] but the most important long term complication is the long term deviation relapse [11].

Different factors are involved in the genesis of the reoccurrence. Besides the clinical features of the primary deviation and the abnormal wide neotendon regrowth in correspondence of the new insertion (microscopic evidence of Schwann cell degeneration both with an increase of the neurofilaments density, axonal vacuoles and hydric swelling $[12,13])$, level variation of some proteins [14] such as fibronectin, proteoglycans, aggrecans and laminin [15] seem to play a basic role in the resected muscles.

The relapse management consist in surgery [16] in- volving lateral rectus or medial rectus (unless previously treated) $[17,18]$.

According to several studies, relapse occurs in about $33 \%$ of cases and it might happen even following redo surgery (although with a lower probability compared with the first surgery $[6,19,20])$.

Most of the extreme eyes deviation $\left(30^{\circ}-45^{\circ}\right.$ or $60^{\Delta}$ $90^{\Delta} \Delta=$ prismatic diopter) evolve to secondary strabismus forms due to recession/resection surgical protocol, extreme anisometropia or surgical trauma [21].

Some surgeons suggested a protocol consisting of a free mucosal transplantation [21] to treat secondary deviations (over $45^{\circ}$ equivalent to $90^{\Delta}$ ).

However, exodeviation surgical correction is still representing a difficult challenge due to the several and different clinical features which make difficult the surgical approach and apparently aleatory outcomes.

\section{Materials and Methods}

The goal of our study was to estimate the effect of a silicone band sutured anteriorly to the lateral rectus neotendon insertion during recession surgery in order to reduce and delay the relapse in the surgical treatment of exotropia.

The study was carried out at the S. Salvatore Hospital of L'Aquila, in the Ophthalmology Unit, from June 2006 to December 2009. Data of 12 patients (8 males and 4 
females) aged between 7 - 54 years with well-known diagnosis of manifest constant exotropia were collected; maximum follow-up at 42 months.

The pre surgical evaluation consisted in anamnesis (in order to evaluate present complaint and previous ocular history); complete ophthalmological assessment; cycloplegic retinoscopy (using tropicamide $1 \%$ and cyclopeantolate $1 \%$ ); best corrected and uncorrected visual acuity check; orthoptic examination (cover-uncover test at both near and distance fixation; ocular motility examination; Worth Lights Test and microWorth test; Titmus Stereo Test and Lang Stereo Test).

The presurgical orthoptic assessment revealed exotropia in all patients; the mean deviation angle at near was $15.4^{\circ}(\mathrm{sd} 1.18)$ and at distance was $13.23^{\circ}$ ( $\left.\mathrm{sd} 0.71\right)$.

The Titmus Test showed absent stereopsis in $50 \%$ of patients (6/12) and a complete perception of circles, with average retinal image disparity of 100 arcs in the remaining $50 \%$ of cases.

Ten over twelve patients (84\%) suppressed one eye with the Worth Lights Test and the remaining $16 \%$ of patients ( 2 over 12$)$ was able to fuse with a proper prismatic correction.

All the procedures were performed by the same experienced surgeon and consisted in $360^{\circ}$ limbal conjuncttival peritomy, medial rectus muscles isolation, lateral rectus recession and silicone band apposition (Novolene 5.0 suture) just anteriorly to the neotendon insertion, layer suture.

\section{Results}

Patients underwent follow up assessment every 6 month; final assessment performed at 42 showed the following results: 8 over 12 patients $(66 \%)$ presented exotropy (mean angle deviation of $3.43^{\circ}$ sd 0.87 ) at near fixation; in the remaining $34 \%$ of cases ( 4 over 12 ) exoforia was detected (mean angle deviation: $4^{\circ}$ sd 0.73 ). Orthophoria for distance was obtained in $25 \%$ of cases (3 patients over 12) and exoforia was still present in 3 over 12 patients $(25 \%)$ (mean angle deviation of $3.42^{\circ}$ sd 0.58 ). In 6 over 12 patients residual small angle exotropia $\left(<1.5^{\circ}\right.$ of deviation). With regards of the sensory evaluation, in $50 \%$ of cases (6 over 12 cases) a modified stereoscopic vision consisnting in perception of the fly, animals and circles was detected, but with a retinal image disparity of 80 arcs. Sensory status check demonstrated suppression in one eye during the test in 10 over 12 cases $(84 \%)$, while in the remaining $16 \%$ of patients $(2 / 12)$ fusion was obtained using an appropriate prismatic correction.

\section{Discussion}

According to several studies, resection/recession surgery produces good outcomes in about $60 \%-80 \%$ of patients
[22] and an early hypercorrection $\left(4^{\Delta}-6^{\Delta}\right)$ [23] seems to represent the only positive predictive factor in the long term success.

Some surgeons proposed to measure intraoperatively the deviated lateral rectus neotendon width in order to estimate the possible functional outcome [24]. A retrospective study involving 350 patients surgically treated for exotropia demonstrated that a good long term outcome (outbreak relapse mean time $=48$ months [25] and 64 months [11]) could be achieved performing a postsurgical hypercorrection higher than $10^{\Delta}$ of isotropia [25].

Hypercorrections higher than $17^{\Delta}$ are often related with consequent secondary isotropia; in these cases, the greatest risk factor [26] is mainly represented by lateral incomitance. A recent study in 2005 showed that hypercorrections higher than $20^{\Delta}$ need a subsequent surgical treatment due to a consequent isotropia in $5 \%, 9 \%$ of the patients [27].

Recently, a 20-year retrospective study involving patients who underwent exotropia surgery before age 10 suggested that stable eye alignment obtained in 11 year old patients tends to be the same until age 30 [28].

The analysis of the results obtained in our study demonstrated a substantial reduction of the mean angle deviation at 42 months after surgery associated with a significant improvement in terms of motor fusion and no presence of flogistic reactions related to silicone band. Stereoscopic sense remained unmodified and the sensory status examination continued to show suppression in one eye. Finally, ocular motility examination didn't suggest any duction disability.

In conclusion, the silicone band in the surgical treatment of manifest exotropia could be a valuable aid in delaying the relapse thanks to its physical and structural features and to its biocompatibility.

\section{REFERENCES}

[1] M. E. Arroyo-Yllanes, D. I. Osorio-Gonzales and J. F. Pèrez-Perèz "Clinical Classification of Variable Angle Exotrophy," Cirugia Cirujanas, Vol. 75, No. 1, 2007, pp. 3-6.

[2] H. E. Willshaw and J. Keenan, "Strabismus Surgery in Children: The Prospects for Binocular Single Vision," Eye, Vol. 5, No. 3, 1991, pp. 338-343. doi:10.1038/eye.1991.54

[3] H. J. Paik and H. B. Yim, "Clinical Effect of Early Surgery in Infantile Exotropia," Korean Journal of OphthaImology, Vol. 16, No. 2, 2002, pp. 97-102.

[4] A. Spierer and G. J. Ben-Simon, "Unilateral and Bilateral Lateral Rectus Recession in Exotropia," Ophthalmic Surgery, Lasers \& Imaging, Vol. 36, No. 2, 2005, pp. 114 117.

[5] L. B. Nelson, D. A. Bacal and M. J. Burke, "An Alternative Approach to the Surgical Management of Exotro- 
pia-The Unilateral Lateral Rectus Recession," Journal of Pediatric Ophthalmology and Strabismus, Vol. 29, No. 6, 1992, pp. 357-360.

[6] .I. R. Hahm, S. W. Yoon, S. H. Baek and S. M. Kong, "The Clinical Course of Recurrent Exotropia after Reoperation for Exodeviation," Korean Journal of Ophthalmology, Vol. 19, No. 2, 2005, pp. 140-144. doi:10.3341/kjo.2005.19.2.140

[7] L. M. Khazaeni and N. J. Volpe, "Adjustable Medial Rectus Muscle Resection in Adult Exotropia," Journal of Pediatric Ophthalmology and Strabismus, Vol. 43, No. 4, 2006, pp. 225-229.

[8] J. W. Jeoung, M. J. Lee and J. M. Hwang, "Bilateral Lateral Rectus Recession versus Unilateral Recess-Resect Procedure for Exotropia with a Dominant Eye," American Journal of Ophthalmology, Vol. 141, No. 4, 2006, pp. 683-688. doi:10.1016/j.ajo.2005.11.021

[9] G. Lennerstrand, "Effects of Surgery on the Dominant Eye in Exodeviations," Acta Ophthalmologica, Vol. 64, No. 4, 1986, pp. 391-396. doi:10.1111/j.1755-3768.1986.tb06941.x

[10] N. K. Koo, Y. C. Lee and S. Y. Lee, "Clinical Study for the Undercorrection Factor in Intermittent Exotropia," Korean Journal of Ophthalmology, Vol. 20, No. 3, 2006 pp. 182-187. doi:10.3341/kjo.2006.20.3.182

[11] S. H. Stoller, J. W. Simon and L. L. Lininger, "Bilateral Lateral Rectus Recession for Exotropia: A Survival Analysis," Journal of Pediatric Ophthalmology and Strabismus, Vol. 31, No. 2, 1994, pp. 89-92.

[12] S. H. Kim, Y. A. Cho, C. H. Park and C. S. Uhm, "The Ultrastructural Changes of Tendon Axonal Profiles of Medial Rectus Muscles According to Duration in Patients with Intermittent Exotropia," Eye, Vol. 22, 2008, pp. 1076-1081. doi:10.1038/eye.2008.133

[13] S. H. Kim, S. T. Yi, Y. A. Cho and C. S. Uhm, "Ultrastructural Study of Extraocular Muscle Tendon Axonal Profiles in Infantile and Intermittent Exotropia," Acta Ophthalmologica Scandinavica, Vol. 84, No. 2, 2006, pp. 182-187. doi:10.1111/j.1600-0420.2005.00556.x

[14] M. Kitada, T. Matsuo, T. Yamane, S. Hasebe and H. Ohtsuki, "Different Levels of Timps and Mmps in Human Lateral and Medial Rectus Muscle Tissue Excised from Strabismic Patients," Strabismus, Vol. 11, No. 3, 2003, pp. 145-155. doi:10.1076/stra.11.3.145.16647

[15] T. Yamane, T. Matsuo, S. Hasebe and H. Ohtsuki, "Clinical Correlations of Aggrecan in the Resected Medial Rectus Muscle of Patients with Intermittent Exotropia," Acta Medica Okayama, Vol. 57, No. 4, 2003, pp. 199204.

[16] Z. Yazdian and G. Ghiassi, "Re-Recession of the Lateral
Rectus Muscles in Patients with Recurrent Exotropia," Journal of AAPOS, Vol. 10, No. 2, 2006, pp. 164-167. doi:10.1016/j.jaapos.2005.11.014

[17] K. I. Chun and S. H. Rah, "The Comparison of Outcomes between Lateral Rectus Muscles Re-Recession and Medial Rectus Muscles Resection in Recurrent Exotropia," Korean Journal of Ophthalmology, Vol. 22, No. 2, 2008, pp. 111-114. doi:10.3341/kjo.2008.22.2.111

[18] J. L. Mims, "Outcome of $5 \mathrm{~mm}$ Resection of One Medial Rectus Extraocular Muscle for Recurrent Exotropia," Binocular Vision \& Strabismus Quarterly, Vol. 18, No. 3, 2003, pp. 143-150.

[19] W. W. Binion, "The Surgical Treatment of Intermittent Exotropia," American Journal of Ophthalmology, Vol. 13, No. 2, 1966, pp. 97-104.

[20] A. Chia, L. Seenyen and Q. B. Long, "A Retrospective Review of 287 Consecutive Children in Singapore Presenting with Intermittent Exotropia," Journal of AAPOS, Vol. 9, No. 3, 2005, pp. 257-263.

[21] W. Lange and S. Motz, "Free Mucosa Transplant in the Correction of Extreme Deviations of Exotropia," Ophthalmologe, Vol. 98, No. 10, 2001, pp. 968-971.

[22] J. E. Berland, M. E. Wilson and R. B. Saunders, "Results of Large (8-9 mm) Bilateral Lateral Rectus Muscle Recessions for Exotropia," Binocular Vision \& Strabismus Quarterly, Vol. 13, No. 2, 1998, pp. 97-104.

[23] T. W. Kim, J. H. Kim and J. M. Hwang, "Surgical Overcorrection Squint Angle in Extropia Related to Retinal Correspondence Relationship," Medicinshi Arhiv, Vol. 61, No. 2, 2007, pp. 94-96.

[24] S. H. Kim and Y. J. Choi, "Effects of Unilateral Lateral Rectus Recession According to the Tendon Width in Intermittent Exotropia," Eye, Vol. 20, No. 7, 2006, pp. 785788.

[25] J. Y. Oh and J. M. Hwang, "Survival Analysis of 365 Patients with Exotropia after Surgery," Eye, Vol. 20, No. 11, 2006, pp. 1268-1272.

[26] H. S. Kim, Y. W. Suh, S. H. Kim and Y. A. Cho, "Consecutive Esotropia in Intermittent Exotropia Patients with Immediate Postoperative Overcorrection More than 17 Prism Diopters," Korean Journal of Ophthalmology, Vol. 21, No. 3, 2007, pp. 155-158.

[27] T. W. Kim, J. H. Kim and J.-M. Hwang "Long-Term Outcome of Patients with Large Overcorrection Following Surgery for Exotropia," Ophthalmologica, Vol. 219, No. 4, 2005, pp. 237-242.

[28] J. J. D. Baker, "Twenty-Year Follow-Up of Surgery for Intermittent Exotropia," Journal of AAPOS, Vol. 12, No. 3, 2008, pp. 227-32. doi:10.1016/j.jaapos.2008.02.007 